\title{
The combination of computed tomography measurements and flexible video bronchoscope guidance for accurate placement of the right-sided double-lumen tube: a randomized controlled trial
}

\section{Yongheng Hou}

First Affiliated Hospital of Soochow University

Huayue Liu

First Affiliated Hospital of Soochow University

Wencheng Shi

First Affiliated Hospital of Soochow University

Hengjing Zhao

First Affiliated Hospital of Soochow University

Ke Peng

First Affiliated Hospital of Soochow University

Hao Cheng

First Affiliated Hospital of Soochow University

Fuhai Ji

First Affiliated Hospital of Soochow University

Jianping Yang ( $\nabla$ szyangjp@126.com )

First Affiliated Hospital of Soochow University

Research article

Keywords: computed tomography, flexible video bronchoscope, lung isolation, one-lung ventilation, rightsided double-lumen tube, thoracic surgery

Posted Date: November 24th, 2020

DOl: https://doi.org/10.21203/rs.3.rs-112957/v1

License: (c) (i) This work is licensed under a Creative Commons Attribution 4.0 International License. Read Full License 


\section{Abstract}

Background: Accurate placement of the right-sided double-lumen tube (RDLT) is still challenging. This study aims to explore the feasibility and accuracy of a modified intubation strategy by using a combination of computed tomography measurements and flexible video bronchoscope guidance.

Methods: 108 adults requiring an RDLT for lung isolation were randomly allocated to 2 groups. Conventional fiberoptic bronchoscopy-guided technique was used in the control group. The following specifications applied to the modification group. Firstly, the length of the right main bronchus (RMB-L) and the anteroposterior diameter of RMB were measured in preoperative spiral computed tomography to predict the side and size of the tube; Then, a depth marker was made on RUSCH tube according to the difference between the RMB-L and the length of bronchia cuff $(12 \mathrm{~mm})$; Under the guidance of flexible video bronchoscope, the depth marker should be paralleled with the tracheal carina, and a characteristic white line on the tube should be paralleled with the secondary carina.

Results: Compared with the control group, our modified strategy significantly increased the optimal plus acceptable position rate (76\% vs. $98 \%$, respectively; $P<0.039)$, decreased tube replacement rate ( $80 \%$ vs. $94 \% ; P=0.042)$, shortened the intubation time (101.4 \pm 7.3 vs. $75.2 \pm 8.1$ seconds; $P=0.019)$, and had a lower incidence of transient hypoxemia ( $25 \%$ vs. $6 \% ; P=0.022)$, subglottic resistance $(20 \%$ vs. $6 \% ; P=$ $0.037)$, tracheobronchial injury ( $35 \%$ vs. $13 ; P=0.037)$, and postoperative right upper lobe collapse $(15 \%$ vs. $2 \% ; P=0.059$ ).

Conclusion: These data suggest the superiority of our modified technique compared to the conventional method for RDLT positioning.

Trial registration: Chinese Clinical Trial Registry, ChiCTR1900021676, registered on 5 March 2019.

URL of trial registry: http://www.chictr.org.cn/showprojen.aspx?proj=33189

\section{Background}

At present, a double-lumen tube (DLT) remains the most commonly used device for lung isolation and one-lung ventilation (OLV) especially in our hospital [1, 2]. The application of the left-sided DLT (LDLT) in preference to the right-sided DLT (RDLT) is recommended for most thoracic surgeries because of its greater safety margin for placement [3]. But for some indications, RDLT may be mandatory; and surgeons also tend to require a non-operative sided DLT [4]. Many different strategies have been explored to improve RDLT placement, including classical auscultation [5], fiberoptic bronchoscopy (FOB) guidance technology [6-8], FOB evaluation before intubation [9], newly designed RDLT [10-13], spirometry monitoring [14], contrast-enhanced ultrasound [15], lateral decubitus rotation [16], and so on. However, so far, accurate placement of an RDLT is still a great challenge, as these methods seem to be neither easily mastered nor commercially available. The most clinically utilized method is just a simple FOB guidance technique similar to the LDLT placement. Although it may be acceptable in most situations, the success 
rate of positioning and the effect of OLV are both significantly lower than those of LDLT, especially in patients with chronic respiratory diseases $[2,3]$.

We consider that the key to a successful RDLT intubation is just to determine the depth and angle of the right upper lobe (RUL) ventilatory slot (RUL-VS) of the tube to the RUL bronchus orifice (RUL-BO) of an individual patient [17]. Nowadays, the routine use of preoperative spiral computed tomography (CT) scan and multi-planar reconstruction (MPR) of three-dimensional (3D) tracheobronchial tree could help to predict the size of a DLT and its insertion depth [18-21]. An anatomical feature between RUL-BO and right middle lobe/right lower lobe bronchus carina (RML/RLL carina) has been proposed as a bony marker to guide the angle of insertion [22]. A continuous white line marker along RUSCH® tube has been noted as an indicator during FOB examination [7]. Besides, flexible video bronchoscope (FVB) has facilitated airway observation in endotracheal intubation $[23,24]$.

In this study, we attempt to develop a modified strategy for the size selection and precise positioning of RDLT by using a combination of CT measurements and FVB guidance. It is our perspective that this strategy may be more feasible and could provide a more effective one-lung ventilation than the conventional method.

\section{Methods}

This study was approved by the Medical Ethics Committee of the First Affiliated Hospital of Soochow University (approval No. 2019 - 0223) and written informed consent was obtained from all subjects participating in the trial. The trial was registered before patient enrolment at chictr.org.cn (ChiCTR1900021676, Principal investigator: Yongheng Hou, Date of registration: March 5, 2019). This manuscript adheres to the applicable Consolidated Standards of Reporting Trials (CONSORT) guidelines.

Patients and groups Data source and study population

From June 2019 to October 2019, 108 patients aged 18-75 years with ASA grade I-III requiring an RDLT for OLV in elective video-assisted thoracoscopic surgery (VATS) were included in the study. The exclusion criteria were as follows: (1) no chest spiral CT examination within 1 month before operation; (2) tracheal or right main bronchial tumors; (3) airway distorted or compressed; (4) anticipated difficult intubation (Mallampati grade > 3); (5) tracheotomy history; (6) tracheal bronchus; (7) suspected tuberculosis or systemic infection; (8) History of thoracic surgery.

Before study commencement, randomization was performed by the use of an online random number generator (http://www.randomization.com), with an allocation ratio of 1:1. In the control group, the conventional selection of tube size based on an adult patient's gender and height was used followed by FOB-guided intubation. In the modification group, our improved strategy was selected (described below). All surgical procedures, anesthesia management, and perioperative care were provided by the same medical team. An independent experienced anesthesiologist assessed RDLT placement and airway change of all patients. 


\section{CT measurements}

Chest imaging, including spiral CT scans and X-ray, are routinely performed for lung tumor diagnosis and localization in our hospital. Preoperative images of all patients were retrieved and measured directly from the hospital imaging system (NEUSOFT image diagnosis systems, DongSoft Inc., Shenyang, China). Multi-planar reconstruction (MPR) of a three-dimensional (3D) tracheobronchial tree has already been performed in all examinations. Three windows were displayed, the lung window, the mediastinal window, and the coronal MPR window. An independent anesthesiologist, specially trained by a senior radiologist, measured different structural distances within the imaging system. Each distance was measured three times and represented as the mean of three measurements. The measurement criteria are described below. Right main bronchus - length (RMB-L): In the MPR window, draw a line from the proximal edge of RUL-BO and cross vertically to the inner side of RMB, and then measure the distance between the tracheal carina and the intersection (Fig. 1). Right main bronchus - Transverse diameter (RMB-TD): In the MPR window, draw a mid-vertical line based on the above RMB-L to intersect the lateral side of RMB and measures the distance between the two intersections (Fig. 1). Right main bronchus - Anteroposterior diameter (RMB-AD): In the lung window, the anteroposterior diameter of RMB at the RMB-BO plane was measured (Fig. 2). Cricoid cartilage - Transverse diameter (CC-TD) and Anteroposterior diameter (CC-AD): The anterior and posterior diameters of the cricoid cartilage were measured in the lung window (Fig. 2).

The spiral CT examination and 3D reconstruction may not be convenient in some community hospitals. We measured RMB-L on the chest X-ray of the same patient and compared it with CT measurement. Draw a line from the trachea carina and cross vertically to the right side of RMB, and next draw a parallel line from the proximal margin of the right upper lobe orifice, and then measure the distance between the two lines and record as RMB-Lx (Supplemental digital Fig. 1).

\section{Tube selection}

In our study, the right RUSCH bronchopart ${ }^{\circledR}$ double lumen bronchial tube set (RUSCH $\circledast 116200$, Teleflex medical, Ireland) was initially selected for all patients. According to our improved method, the sizes of RDLT selected were 28F, 35F, 37F, 39F, and 41F for RMB-AD of $<9.0 \mathrm{~mm}, 11.5 \mathrm{~mm}, 12.2 \mathrm{~mm}, 12.9 \mathrm{~mm}$, and $>12.9 \mathrm{~mm}$, respectively (Table 1 ). We noticed that the distance from the proximal end of the bronchial cuff to the proximal end of RUL-VS was designed to be about $12 \mathrm{~mm}$ regardless of different tube sizes. The difference $(X)$ between RMB-L and 12 was calculated if RMB-L $>12 \mathrm{~mm}$. Before intubation, we used a surgical marker pen to draw a black line $X \mathrm{~mm}$ above the distal end of the bronchial cuff on the external wall of the tube (Fig. 1,3). We also noticed a full-length white line marker on the tube, which was designed as an X-ray marker for checking position. Its leading-edge is always paralleled with the midline of RUL-VS (Fig. 2,3). Before use, the endobronchial cuff and endotracheal cuff were prepared with gas leakage measurement, well lubricated with lidocaine cream, and aspirated fully to collapse each balloon [25]. 
Table 1

Brief description of the modified strategy.

\section{Question Answer}

Which Measure RMB-L and RMB-AD in spiral CT or chest X-ray images.

side?

RMB-L $<9 \mathrm{~mm}$ is not suitable for RDLT; Replace with LDLT.

Which

size?

\begin{tabular}{|lll|}
\hline RMB-AD $(\mathrm{mm})$ & Corresponding height $(\mathrm{cm})$ & RDLT size $(\mathrm{F})$ \\
\hline$<9(<9)$ & $<141$ & 28 \\
$9 \sim 11.5(\approx 9 \sim 11)$ & $\approx 141 \sim 160$ & 35 \\
$11.5 \sim 12.2(\approx 11 \sim 12)$ & $\approx 160 \sim 165$ & 37 \\
$12.2 \sim 12.9(\approx 12 \sim 13)$ & $\approx 165 \sim 172$ & 39 \\
$12.9 \sim 13.6(>13)$ & $>172$ & 41 \\
\hline
\end{tabular}

How Make a black maker at (RMB-L minus 12) $\mathrm{mm}$ above bronchial cuff before intubation; deep? Guide the black marker to align with the tracheal carina.

Which Guide the line between white marker and X-ray indicator to align with the RML/RLL angle? carina.

What Check the alignment of the RUL-VS and RUL-BO through the slit of the tube.

result?

$\mathrm{CT}$, computed tomography; DLT, double-lumen tube; FOB, fiberoptic bronchoscopy; FVB, flexible video bronchoscope; LDLT, left-sided double-lumen tube; RDLT, right-sided double-lumen tube; RMB-AD, right main bronchus- anteroposterior diameter; RMB-L, right main bronchus - length ; RML/RLL carina, right middle lobe /right lower lobe bronchus carina; RUL, right upper lobe; RUL-BO, RUL bronchial orifice; RUL-VS, RUL ventilation slot.

\section{Modified intubation of RDLT}

A 3.5-mm diameter electronic portable flexible video bronchoscopy (Seesheen SS-2130, Zhuhai, China) was used in the modification group (Fig. 3). After general anesthesia induction, the RDLT was inserted into the glottis under a visual laryngoscope (E.an-II L, Tianjin, China) $[25,26]$. Once the endobronchial tip of RDLT was passed through the vocal cords, the stylet would be removed, and the tube was rotated $90^{\circ}$ to the right and slightly advanced until resistance was encountered. The FVB was subsequently passed through the tracheal lumen to ensure that the bronchial portion of DLT was in the right bronchus and then to guide the depth of the tube, noting that the aforementioned black line should be paralleled with the tracheal carina. Then, the FVB was withdrawn and reinserted through the bronchial lumen. An aforementioned white line marker could be seen on the left side of the inner wall through the full length of the tube. Insert FVB downward along the white line marker until the top of the tube, RML/RLL orifices, and the bronchial carina could be seen. Bronchial carina should be paralleled with the line between the white marker and the X-ray indicator. A rotational motion may be needed to align the two lines on the condition 
that the cuff is deflated. Next, bend the FVB tip to the right and withdraw slowly to look for the RUL-VS, which was just on the opposite side of the white line marker. Through the RUL-VS slit, we can check the alignment of RUL-VS and RUL-BO. The alignment should be rechecked and repositioned if needed after lateral positioning. A brief description of each option of the strategy is listed in Table 1 for better understanding.

\section{Conventional intubation of RDLT}

In the control group, the selection of RDLT size was based on an adult patient's gender and height [5, 27]. The intubation procedure in the control group was the same as that in the modification group before the step of the FOB (Pentax F19BX, Japan) examination. First, FOB was inserted into the trachea lumen. The inflated blue bronchial balloon should be visible beyond the carina on the left side without herniation into the trachea. Then, FOB was withdrawn and reinserted through the bronchial lumen to search for RUL-VS. When the FOB tip passed through RUL-VS, RUL-BO with three segments should be visible.

\section{Variables and endpoint}

The primary endpoint was the number of positioned RDLT, which is defined as the alignment of the proximal edge of RUL-VS with the proximal edge of RUL-BO (Fig. 1). It is expressed as the visualization score of the RUL-BO: 1, completely visible (the optimal position); 2, partially visible (acceptable position); 3 , completely invisible (wrong position). The secondary endpoints included intubation data and perioperative adverse events. Intubation data: tube size; intubation resistance; cuff pressure; intubation time; intubation failure; visual field score of right bronchial origin under FOB; lung isolation effect. Perioperative adverse events: intraoperative transient hypoxemia $(\mathrm{SpO} 2<90 \%)$, high airway pressure $(>$ $35 \mathrm{cmH}_{2} \mathrm{O}$ ), air leakage (low airway pressure $<10 \mathrm{cmH}_{2} \mathrm{O}$ ), carina, and bronchial injury. Intubation failure: After 3 attempts, the endobronchial lumen could not be inserted into RMB. Intubation resistance: 1, no resistance; 2 , mild resistance; 3 , moderate resistance; 4 , severe resistance, it cannot pass through the subglottis and must be replaced by the next smaller size. The pressure of the bronchial section: The pressure of the bronchial tube was measured by a hand-held pressure gauge (Rush Endotest 112700). When the required pressure of the bronchial sleeve is $0 \mathrm{cmH}_{2} \mathrm{O}$, the bronchial segment is considered too large; when the pressure of the bronchial sleeve is greater than $30 \mathrm{~cm} \mathrm{H}_{2} \mathrm{O}$, the bronchial segment is too small. Lung isolation effect score: 1, excellent (complete collapse); 2 , fair (some residual air); and 3, poor (no collapse or residual air interfering with surgical exposure). Trachea and bronchial injury: 1, obvious; 2 , a small amount of ecchymosis; 3 , combined with ecchymosis, bleeding or ecchymosis; 4 , erosion. The collapse of the right upper lobe was examined by bedside chest radiographs at the post-operative stage.

\section{Statistical analysis}

The sample size was calculated based on previous clinical experience and previous studies. 50 patients in each group were required with a test level of 0.05 and an assurance of $80 \%$. Quantitative variables were represented as means (standard deviation) or medians (25th percentile; 75th percentile) and compared using unpaired t-test or paired t-test, with Welch's correction if required. Mann-Whitney ranksum test was used to analyze the non-parametric data and the score-based results. The qualitative variable was represented by a number (proportion) and compared using Fisher exact test or chi-square 
test, when appropriate. Linear regression analysis was used to evaluate how RMB-L in the CT images related to those in the chest X-ray images. The relationship between $\mathrm{CT}$ measurements and age were also examined using a linear regression approach. The statistical analysis was done by using GraphPad Prism 7.0 (GraphPad Software Inc. San Diego, CA, USA) and a P-value of $<0.05$ was considered statistically significant.

\section{Results}

A total of 108 patients were assessed for eligibility in our study. After exclusions, 100 patients were enrolled and randomized 1:1 to the modification group $(n=50)$ and the control group $(n=50)$. Five patients were excluded in further analysis. Among them, two patients in the control group and 1 patient in the modification group failed to intubate the RDLTs and changed to LDLTs, and the LDLTs were directly used in another 2 patients in the modification group according to the CT measurements (Fig. 4). There were no significant differences in demographic data, ASA grade, Mallampati score, Surgical Procedures, and CT measurements between the two groups (Table 2). 
Table 2

Demographic data and CT measurements data.

\begin{tabular}{|c|c|c|c|}
\hline & $\begin{array}{l}\text { Control group } \\
(n=50)\end{array}$ & $\begin{array}{l}\text { Modification group } \\
(n=50)\end{array}$ & $P$ Value \\
\hline \multicolumn{4}{|l|}{ Demographic data } \\
\hline Age (y) & $58.1(1.6)$ & $53.5(1.9)$ & 0.246 \\
\hline Height (cm) & $162.9(1.1)$ & $163.8(1.3)$ & 0.208 \\
\hline Weight (kg) & $63.9(1.7)$ & $61.5(1.8)$ & 0.735 \\
\hline ASA, $n$ & & & 0.369 \\
\hline I & $10(20 \%)$ & $12(24 \%)$ & \\
\hline II & $36(72 \%)$ & $37(74 \%)$ & \\
\hline III & $4(8 \%)$ & $1(2 \%)$ & \\
\hline Gender, n & & & 0.548 \\
\hline M & $25(50 \%)$ & $21(42 \%)$ & \\
\hline $\mathrm{F}$ & $25(50 \%)$ & $29(58 \%)$ & \\
\hline Mallampati score, $\mathrm{n}$ & $20(40 \%)$ & $18(36 \%)$ & 0.837 \\
\hline 1 & $30(60 \%)$ & $32(64 \%)$ & \\
\hline \multicolumn{4}{|l|}{2} \\
\hline Surgical Procedures, $\mathrm{n}$ & & & 0.414 \\
\hline Wedge & $10(20 \%)$ & $12(24 \%)$ & \\
\hline Segmental & $2(4 \%)$ & $2(4 \%)$ & \\
\hline Upper left lobectomy & $17(34 \%)$ & $9(18 \%)$ & \\
\hline Lower left lobectomy & $13(26 \%)$ & $14(28 \%)$ & \\
\hline Pneumonectomy & $8(16 \%)$ & $13(26 \%)$ & \\
\hline \multicolumn{4}{|c|}{$\mathrm{CT}$ or X-ray measurements $(\mathrm{mm})$} \\
\hline RMB-L & $15.4(3.9)$ & $14.9(3.8)$ & 0.109 \\
\hline
\end{tabular}

Values are mean (SD), number (proportion).

Abbreviations: $\mathrm{BMI}$, body mass index; $\mathrm{CC}-\mathrm{AD}$, cricoid cartilage - anteroposterior diameter; CC-TD, cricoid cartilage - transverse diameter; $F$, female; $M$, male; $R M B-A D$, right main bronchus anteroposterior diameter; RMB-L, right main bronchus - length; RMB-Lx, RMB-L in chest X-ray image; RMB-TD, right main bronchus - transverse diameter. 


\begin{tabular}{|llll|}
\hline & $\begin{array}{l}\text { Control group } \\
(\mathbf{n = 5 0 )}\end{array}$ & $\begin{array}{l}\text { Modification group } \\
(\mathbf{n = 5 0 )}\end{array}$ & PValue \\
\hline RMB-Lx & $14.9(8.6)$ & $14.2(9.8)$ & 0.143 \\
\hline RMB-TD & $13.9(2.0)$ & $13.7(2.4)$ & 0.569 \\
\hline RMB-AD & $12.7(2.1)$ & $12.3(2.1)$ & 0.379 \\
\hline CC- TD & $16.5(3.1)$ & $16.6(2.7)$ & 0.905 \\
\hline CC- AD & $15.5(3.5)$ & $15.9(3.6)$ & 0.484 \\
\hline Values are mean (SD), number (proportion). & & \\
\hline $\begin{array}{l}\text { Abbreviations: BMI, body mass index; CC-AD, cricoid cartilage - anteroposterior diameter; CC-TD, } \\
\text { cricoid cartilage - transverse diameter; F, female; M, male; RMB-AD, right main bronchus - } \\
\text { anteroposterior diameter; RMB-L, right main bronchus - length; RMB-Lx, RMB-L in chest X-ray image; } \\
\text { RMB-TD, right main bronchus - transverse diameter. }\end{array}$ & \\
\hline
\end{tabular}

CT measurements of the two groups were mixed together for further analysis. We found that RMB-L ranged from $7.15 \mathrm{~mm}$ to $25.67 \mathrm{~mm}$, with an average of $15.1 \pm 3.93 \mathrm{~mm}$. Among them, $6 \%$ were $>22 \mathrm{~mm}$, $77 \%$ were $12-22 \mathrm{~mm}, 12 \%$ were $9-12 \mathrm{~mm}$ and $5 \%$ were $<9 \mathrm{~mm}$ (too short for an RDLT). No significant correlations were found between RMB-L and gender $(P=0.2547)$ or Height $(P=0.8222)$, respectively. From the alternative measurement in chest X-ray, the mean (SD) of RMB-Lx was 14.6 (8.78) $\mathrm{mm}$. The inter-observer agreement was substantial with both methods (CT: interclass correlation coefficient [ICC] = 0.84 ; X-ray: ICC $=0.95)$. Correlations were strong and significant for RMB-L in CT $(r=0.72, P<0.01)$ and in $X$-ray $(r=0.60, P<0.01)$. Other measurement results are presented as mean $\pm S D$ ( $m a x, \min )$ and listed as follows: CC-TD, $16.54 \pm 2.865$ (14.59-24.03) mm; CC-AD, $15.71 \pm 3.594$ (12.87-24.33) mm; RMB-TD, $13.82 \pm 2.176(12.48-19.25) \mathrm{mm}$; RMB-AD, $12.47 \pm 2.128(10.94-19.25) \mathrm{mm}$. It was found that RMB-AD was the smallest $(P<0.0001)$, suggesting that it could be suitable as a criterion for tube size selection. In general, there was a linear correlation between RMB-AD and height $\left(R^{2}=.2727, P<0.0001\right)$ : RMB-AD $(\mathrm{mm})=0.133^{*}$ height $(\mathrm{cm})-9.882$.

The intubation data are shown in Table 2. According to the conventional method or our modified method, the final selection of tube size has no statistical significance in proportion. RDLTs in 8 patients of the control group needed to be replaced with larger or smaller RDLTs after the first attempt. For another 2 patients, the LDLTs have to be used because of severe air leakage. FVB/FOB examination showed that the bronchial cuff partially exfoliated outside the carina. However, in the modification group, only one case needed to be replaced with a smaller tube. After consulting with the surgeon, the LDLTs were directly selected for 3 patients according to their RMB-L. The time spent on intubation in the modification group was statistically shorter than that in the control group $(75.2 \pm 8.1$ vs $101.4 \pm 7.3$ seconds; $P=0.0186)$, although it may lack clinical significance in the context of surgery. A significant difference was found in subglottic resistance between the two groups $(P=0.0293)$. The accuracy of the bronchial segment size 
selection was similar between the two groups $(P=0.1220)$. In the control group, 21 of 48 cases were in the optimal position (43\%), 16 (33) in acceptable position, and $9(19 \%)$ in the wrong position. In the modification group, 31 of $47(77 \%)$ cases could be were in the optimal position, 10 (21\%) were in the acceptable position, and only 1 (2\%) was completely invisible to RUL-BO. The difference was statistically significant in optimal plus acceptable position between the two groups ( $62 \%$ vs $98 \%, P=0.0388)$. There was no significant difference in lung isolation scores between the two groups $(P=0.6018)$.

Perioperative adverse events data are listed in Table 4. In the control group, the incidence of transient hypoxemia during OLV was $25 \%$, while only 3 cases (8.5\%) had hypoxemia in the modification group. The incidences of airway hypertension and hypotension during OLV and airway injury were significantly lower in the modification group than in the control group $(P=0.0104$ and $P=0.0357)$. Right upper lobe collapse was reported postoperatively in 5 patients in the control group and only one patient in the modification group by bedside chest $\mathrm{X}$-ray $(P=0.0590)$. 
Table 4

Perioperative adverse events.

\begin{tabular}{|c|c|c|c|}
\hline & $\begin{array}{l}\text { Control group } \\
(n=48)\end{array}$ & $\begin{array}{l}\text { Modification group } \\
(n=47)\end{array}$ & $P$ Value \\
\hline Transient Hypoxemia, n (\%) & & & 0.0221 \\
\hline Yes & $12(25)$ & $3(6)$ & \\
\hline No & $36(75)$ & $44(94)$ & \\
\hline Airway pressure, n (\%) & & & 0.0104 \\
\hline High (> 35 cmH2O) & $14(29)$ & $5(11)$ & \\
\hline Low $(<10 \mathrm{cmH} 20)$ & $8(17)$ & $3(6)$ & \\
\hline Normal & $26(54)$ & $39(83)$ & \\
\hline Tracheobronchial injury, n (\%) & & & 0.0357 \\
\hline Clear & $31(65)$ & $41(87)$ & \\
\hline A few petechiae & $12(25)$ & $5(11)$ & \\
\hline Coalesced petechiae, hemorrhage, or ecchymosis & $5(10)$ & $2(4)$ & \\
\hline Erosion & $0(0)$ & $0(0)$ & \\
\hline Postoperative RUL collapse, n (\%) & & & 0.0490 \\
\hline Yes & $7(15)$ & $1(2)$ & \\
\hline No & $41(85)$ & $46(98)$ & \\
\hline \multicolumn{4}{|l|}{ Values are mean (SD), number (proportion). } \\
\hline $\begin{array}{l}\text { Abbreviations: DLT, double-lumen tube; LDLT, left-si } \\
\text { RDLT, Right-sided DLT; RUL, right upper lobe. }\end{array}$ & double-lum & abe; OLV, one-lung & lation; \\
\hline
\end{tabular}

\section{Discussion}

RDLT always had a bad reputation for being difficult to positioning because of its small margin of safety, and so its clinical use was rigorously avoided by many experts in the field of thoracic anesthesia [3]. However, in several certain select cases, the use of an RDLT may be mandatory [7]. In our history of clinical practices, there were several accidents that LDLT was sutured to the incision of left sleeve bronchotomy or pneumonectomy, so the bronchial intubation on the non-surgical side is preferred by our thoracic surgeons whenever possible. Many suggestions have been proposed to improve RDLT placement, but are poorly effective. Currently, FOB guidance remains the gold standard for verification of 
the DLT position [28]. And FVB, as a portable electronic bronchoscope, is an alternative method for airway observation [23]. Its design of a high-definition screen mounted on the eyepiece could provide a more detailed picture of the bronchial structure, which is very helpful to the anesthesiologists in DLT placement.

As for the selection of ideal DLT size, it is generally accepted that it should be based on the principle of smoothly inserting the largest type of tube into the target bronchus $[29,30]$. Conventionally, the size selection is based on a person's height and gender, which was also adopted in the control group [5]. Although clinically acceptable in most cases, it may be not always appropriate especially for older adults. According to a recent study, the optimal size of the DLT should be selected based on the combination of the transverse diameter of the cricoid ring and the main bronchus [31, 32]. In our study, cricoid cartilage was chosen as the measurement plane of tracheal diameter because its diameter is considered the smallest in the whole trachea [33]. The RUL-BO plane was chosen to measure RMB diameter because the key to a successful RDLT is just to ensure RUL ventilation. As for the tube, the diameter of the tracheal part other than the bronchial part of RDLT was chosen to match with RMB-AD, because the tracheal part is often inserted too deep into the bronchus during intubation. Benumof et al.[22] have shown that the ideal bronchial diameter of DLT should be 1 to $2 \mathrm{~mm}$ smaller than that of the patient's main bronchi. We found that RMB-AD was the smallest in all CT measurements and had a significant linear correlation with the height regardless of different genders, suggesting that it could be a suitable criterion for tube size selection [31]. The corresponding heights calculated according to the formula were consistent with conventional selection (Table 1) [2]. In the modification group, almost all selected DLTs were appropriate in the bronchial segment with a lower intubation resistance and could significantly reduce the rate. The incidence of tube replacement and the poor airway pressure change during OLV in the modification group was significantly lower than that in the control group. Besides, our method can effectively prevent tracheal and bronchial injury without sacrificing the effectiveness of lung separation.

Estimating RMB-L is of paramount importance for a successful RDLT. Benumof et al.[34] defined the margin of safety of Rusch RDLT intubation as the length of the RUL-VS minus RUL diameter. Kim JH et al.[17, 35] suggested that LDLT should be considered if the distance between the tracheal carina and the distal edge of the RUL bronchus is less than $23 \mathrm{~mm}$. However, the above studies did not take into account the design and size of different brands of DLTs. Even Rusch DLT's design has changed greatly from 30 years ago [34]. We define RMB-L as the distance from the tracheal carina to the proximal edge of the RUL-BO because it is the easiest to be distinguished and measured in spiral CT(Fig. 1). After a comprehensive analysis of the CT measurements of 100 Chinese patients in the two groups, we found that the RMB-L ranges from $7.15 \mathrm{~mm}$ to $25.67 \mathrm{~mm}$ with an average of $15.1 \pm 3.93$. No abnormal tracheal origin of the RUL bronchus was found in all subjects. Because of the inconsistency of the measurement methods, the results were slightly longer than those of Mi et al.[20] (13.6 \pm 4.3$)$. The RMB-L showed greater individual variability in Chinese but had a statistically significant positive correlation with height regardless of gender. The measurement results from the chest X-ray are also acceptable. There is no significant difference between the two methods, except that sometimes the anatomical structure may not develop well. 
We noticed that the distance from the proximal end of the bronchial cuff to the proximal end of RUL-VS in a standard Rusch tube is always about $12 \pm 0.5 \mathrm{~mm}$. But, the length of RUL-VS of different tubes varied considerably from 8 to $15 \mathrm{~mm}$, suggesting that it is not suitable as an indicator for intubation. If the alignment of the bronchial cuff with tracheal carina is taken as standard, the optimal RMB-L should be about $12 \mathrm{~mm}$. In fact, in the cases of RMB-L 9-12 mm (12\%), the bronchial cuff can be partially located in the trachea without leakage, thus increasing the safety range. Therefore, we suggest that Rusch RDLT is inapplicable to the patients with RMB-L less than $9 \mathrm{~mm} \mathrm{(5 \% )}$ and the right-sided intubation will inevitably be a failure. When RMB-L is longer than $22 \mathrm{~mm} \mathrm{(6 \% )}$ and the bronchial cuff is paralleled with tracheal carina, it could lead to complete blockage. For most patients in the control group (77\%) with RMB-L 12-22 mm, effective lung isolation was clinically acceptable without severe hypoxemia as long as RUL-VS is partially paralleled with RUL-BO. Yet we think that we can do even better. We suggest that the optimal depth for an RDLT should be the alignment of the proximal end of RUL-VS with the proximal end of the RUL-BO to ensure the most effective ventilation. Since we have already known RMB-L, we can calculate the difference between RMB-L and 12, and then make a black marker there on the outer wall of the tube before intubation. Under the guidance of FVB, the depth of the right upper lung can be easily and accurately located only by adjusting the relative position of the black marker to the tracheal carina.

The ideal insertion angle should be considered after the optimal depth is determined. Campos JH et al. [13] observed that RDLT must be rotated in some patients to obtain the correct alignment of the endobronchial tube with the RUL-BO. We noticed that the RUL-BO is always directly identical to RML/RLL carina. This structural feature can be seen through CT imaging and 3D reconstruction. We also noticed a full-length white line marker on the inner surface of the whole tube. Campos $\mathrm{JH}$ et al. [7] suggested that the side hole is just opposite the white line, which can be used as a sign of intubation. Further observation shows that the front end of the white line marker is precisely paralleled with the middle line of RUL-VS. Therefore, the theoretical optimal insertion angle can be easily achieved by keeping the two lines in parallel (Fig. 2). Our results showed that the number of cases with the best position was significantly superior in the modification group to that in the control group. Although our method seems a little complex, the intubation time is significantly shortened, although it may lack clinical significance in the context of surgery (Table 3 ). 
Table 3

Intubation data.

\begin{tabular}{|c|c|c|c|}
\hline & $\begin{array}{l}\text { Control group } \\
(n=50)\end{array}$ & $\begin{array}{l}\text { Modification group } \\
(n=50)\end{array}$ & $P$ Value \\
\hline Intubation time, s & $101.4 \pm 7.3$ & $75.2 \pm 8.1$ & 0.0186 \\
\hline DLT size selected, $\mathrm{n}(\%)$ & & & 0.9850 \\
\hline $41 \mathrm{~F}$ & $3(6)$ & $3(6)$ & \\
\hline $39 \mathrm{~F}$ & $6(12)$ & $8(16)$ & \\
\hline $37 \mathrm{~F}$ & $14(28)$ & $12(24)$ & \\
\hline $35 \mathrm{~F}$ & $23(46)$ & $22(44)$ & \\
\hline $28 \mathrm{~F}$ & $2(4)$ & $2(4)$ & \\
\hline Replacement of RDLT, n (\%) & & & 0.0415 \\
\hline Appropriate & $40(80)$ & $47(94)$ & \\
\hline with the next smaller size & $4(8)$ & $1(2)$ & \\
\hline with the next larger size & $4(8)$ & $0(0)$ & \\
\hline with LDLT & $2(4)$ & $3(6)$ & \\
\hline Resistance at the subglottic, $\mathrm{n}(\%)$ & & & 0.0293 \\
\hline None & $25(50)$ & $38(76)$ & \\
\hline Mild & $15(30)$ & $6(12)$ & \\
\hline Moderate & $4(8)$ & $2(4)$ & \\
\hline Severe & $4(8)$ & $1(2)$ & \\
\hline Bronchial segment size selected, n (\%) & & & 0.1220 \\
\hline Oversized & $3(6)$ & $1(2)$ & \\
\hline Correct & $38(76)$ & $44(88)$ & \\
\hline Undersized & $7(14)$ & $2(4)$ & \\
\hline Visualization of RUL-BO, n (\%) & & & 0.0388 \\
\hline Complete (optimal position) & $21(43)$ & $36(77)$ & \\
\hline
\end{tabular}

Values are mean (SD), number (proportion).

Abbreviations: DLT, double-lumen tube; LDLT, left-sided double-lumen tube; OLV, one-lung ventilation; RDLT, Right-sided DLT; RUL, right upper lobe; RUL-BO, RUL bronchial orifice. 


\begin{tabular}{|llll|}
\hline & $\begin{array}{l}\text { Control group } \\
(\mathbf{n = 5 0 )}\end{array}$ & $\begin{array}{l}\text { Modification group } \\
(\mathbf{n = 5 0 )}\end{array}$ & PValue \\
\hline Partial (acceptable position) & $16(33)$ & $10(21)$ & \\
\hline Absence (wrong position) & $9(19)$ & $1(2)$ & 0.6018 \\
\hline Left lung collapse in OLV, $\mathrm{n}(\%)$ & $30(63)$ & $28(60)$ & \\
Complete & $14(29)$ & $17(36)$ & \\
Partial & $4(8)$ & $2(4)$ & \\
None & & & \\
\hline Values are mean (SD), number (proportion). & & \\
\hline Abbreviations: DLT, double-lumen tube; LDLT, left-sided double-lumen tube; OLV, one-lung ventilation; \\
\hline RDLT, Right-sided DLT; RUL, right upper lobe; RUL-BO, RUL bronchial orifice.
\end{tabular}

Hypoxemia during OLV is the most common complication of DLT intubation. The best prevention and treatment is a reliable positioning as accurately as we can. Ehrenfeld JM et al. [36] thought that anesthesiologists, even infrequent users, could place RDLTs as reliably as LDLT with the same incident of intraoperative hypoxia, hypercapnia, and high airway pressures. We found a $25 \%$ incidence of transient or persistent hypoxemia in the control group, but it could be easily treated in in the vast majority of cases [2]. Bronchoscopy examination showed that most of the tube was in a sub-location state, although their $\mathrm{SpO} 2$ values and OLV effect may be acceptable in most situations. Our improved method could significantly reduce the incidence of transient hypoxemia and thus improve the efficiency of general anesthesia. Airway trauma and trachea-bronchial membranes rupture are potential complications of DLT. Airway trauma can occur when a small-sized DLT moves from a larger-than-sized DLT to a distal lobar bronchus. Increased pressure exerted by the envelope on the mucosa is also a potential cause of tissue damage. In this study, the incidence of airway injury was significantly lower in the modification group than in the control group by minimizing the need for adjusting the tube repeatedly. Hoarseness and sore throat are also common complications [31]. In our hospital, acupoint application has been widely used to effectively prevent post-operative pharyngalgia, so this article does not cover these contents [37].

This study has some limitations. Firstly, there may be some potential biases during CT measurements. Secondly, the white line marker in RUSCH® tube, which plays an important role in our strategy, did not appear in other commercial designs of RDLT. However, the same effect can be easily realized by drawing a self-made marker on the top of a domestic tube (Haisheng® DLT3202, Zhejiang, China) (Supplemental digital Fig. 2). Lastly, the method also needs to be repeated by other groups to proves its value and make further improvements, especially in European and American populations.

\section{Conclusions}


In conclusion, our modified strategy could easily, safely, and accurately determine RDLT placement by combining CT measurements with FVB guidance, which may be an alternative approach to the conventional method.

\section{Abbreviations}

CC-TD, Transverse diameter of cricoid cartilage;

CC-AD, Transverse diameter of cricoid cartilage

CT, computed tomography;

DLT, double-lumen tube;

FOB, fiberoptic bronchoscopy

FVB, flexible video bronchoscope;

LDLT, Left-sided DLT;

RDLT, Right-sided DLT;

OLV, one-lung ventilation;

$\mathrm{RMB}$, right main bronchus;

RMB-L, length of right main bronchus;

RMB-TD, Transverse diameter of right main bronchus;

RMB-AD, Anteroposterior diameter of right main bronchus;

$\mathrm{RML} / \mathrm{RLL}$ carina, right middle lobe /right lower lobe bronchus carina;

RUL, right upper lobe;

RUL-BO, RUL bronchial orifice;

RUL-VS, RUL ventilation slot;

VATS, video-assisted thoracic surgery.

\section{Declarations}

Ethics approval and consent to participate 
This study was approved by the Medical Ethics Committee of the First Affiliated Hospital of Soochow University (approval No. 2019-0223) and written informed consent was obtained from all subjects participating in the trial. The trial was registered before patient enrolment at chictr.org.cn (ChiCTR1900021676, Principal investigator: Yongheng Hou, Date of registration: March 5, 2019). This manuscript adheres to the applicable Consolidated Standards of Reporting Trials (CONSORT) guidelines.

\section{Consent for publication}

Not applicable.

\section{Availability of data and materials}

The datasets used and/or analyzed during the current study are available from the corresponding author on reasonable request.

\section{Competing interests}

The authors declare that they have no competing interests.

\section{Funding}

No funding was obtained for this study.

\section{Authors' Contributions}

YH designed and performed the study, interpreted and analyzed the data, performed statistical analyses, and wrote and revised the manuscript. JY designed the study, interpreted and analyzed the data, and revised the manuscript. KP, WS, and $\mathrm{HZ}$ collected data and wrote and revised the manuscript. $\mathrm{HC}$ and $\mathrm{FJ}$ revised the manuscript critically for important intellectual content. All authors approved the final manuscript.

\section{Acknowledgements}

Not Applicable

\section{References}

1 Campos $\mathrm{JH}$ : Which device should be considered the best for lung isolation: double-lumen endotracheal tube versus bronchial blockers. Curr Opin Anaesthesiol 2007;20:27-31.

2 SLINGER PD, CAMPOS JH: Miller's anesthesia, vol. 2. 8th ed., Elsevier Health Sciences: Benjamin, 2014.

3 Cohen E: Con: right-sided double-lumen endotracheal tubes should not be routinely used in thoracic surgery. J Cardiothorac Vasc Anesth 2002;16:249-252. 
4 Pedoto A: How to choose the double-lumen tube size and side: the eternal debate. Anesthesiol Clin 2012;30:671-681.

5 SLINGER PD, CAMPOS JH: Anesthesia for thoracic surgery.; in Miller RD (ed Miller's Anesthesia. 8th ed. Philadelphia, Elsevier Churchill Livingstone, 2014, pp 1942-2005.

6 Alliaume B, Coddens J, Deloof T: Reliability of auscultation in positioning of double-lumen endobronchial tubes. Can J Anaesth 1992;39:687-690.

7 Campos JH, Gomez MN: Pro: right-sided double-lumen endotracheal tubes should be routinely used in thoracic surgery. J Cardiothorac Vasc Anesth 2002;16:246-248.

8 Klein U, Karzai W, Bloos F, Wohlfarth M, Gottschall R, Fritz H, Gugel M, Seifert A: Role of fiberoptic bronchoscopy in conjunction with the use of double-lumen tubes for thoracic anesthesia: a prospective study. ANESTHESIOLOGY 1998;88:346-350.

9 Amin N, Tarwade P, Shetmahajan M, Pramesh CS, Jiwnani S, Mahajan A, Purandare N: A randomized trial to assess the utility of preintubation adult fiberoptic bronchoscope assessment in patients for thoracic surgery requiring one-lung ventilation. Ann Card Anaesth 2016;19:251-255.

10 Bussieres JS, Lacasse Y, Cote D, Beauvais M, St-Onge S, Lemieux J, Soucy J: Modified rightsided broncho-cath double lumen tube improves endobronchial positioning: a randomized study. Can J Anaesth 2007; 54:276-282.

11 Hagihira S, Takashina M, Mashimo T: Application of a newly designed right-sided, double-lumen endobronchial tube in patients with a very short right mainstem bronchus. ANESTHESIOLOGY 2008;109:565-568.

12 Mercier FJ, Fischler M: Is it possible to improve the shape of right double-lumen endobronchial tubes? J Cardiothorac Vasc Anesth 1995;9:236.

13 Campos JH, Massa FC, Kernstine KH: The incidence of right upper-lobe collapse when comparing a right-sided double-lumen tube versus a modified left double-lumen tube for left-sided thoracic surgery. ANESTH ANALG 2000;90:535-540.

14 Ye J, Ouyang BY, Dong QL: [estimation of the position of right-sided double-lumen endobronchial tubes with spirometry in elderly patients]. Nan Fang Yi Ke Da Xue Xue Bao 2009;29:469-471.

15 Hu WC, Xu L, Zhang Q, Wei L, Zhang W: Point-of-care ultrasound versus auscultation in determining the position of double-lumen tube. Medicine (Baltimore) 2018;97:e9311.

16 Yu W, Wang Z, Gao D, Zhang W, Jin W, Ma X, Qi S: A method for addressing right upper lobe obstruction with right-sided double-lumen endobronchial tubes during surgery: a randomized controlled trial. BMC ANESTHESIOL 2018;18:130. 
Bussieres JS, Gingras M, Perron L, Somma J, Frenette M, Couture EJ, Moreault O, Lacasse Y:

Right upper lobe anatomy revisited: a computed tomography scan study. Can J Anaesth 2019;66:813819.

18 Liu Z, Zhao L, Jia Q, Yang X, Liang SJ, He W: Chest computed tomography image for accurately predicting the optimal insertion depth of left-sided double-lumen tube. $J$ Cardiothorac Vasc Anesth 2018;32:855-859.

19 Hannallah M, Benumof JL, Silverman PM, Kelly LC, Lea D: Evaluation of an approach to choosing a left double-lumen tube size based on chest computed tomographic scan measurement of left mainstem bronchial diameter. J Cardiothorac Vasc Anesth 1997;11:168-171.

20 Mi W, Zhang C, Wang H, Cao J, Li C, Yang L, Guo F, Wang X, Yang T: Measurement and analysis of the tracheobronchial tree in chinese population using computed tomography. PLOS ONE 2015;10:e123177.

21 Eberle B, Weiler N, Vogel N, Kauczor HU, Heinrichs W: Computed tomography-based tracheobronchial image reconstruction allows selection of the individually appropriate double-lumen tube size. J Cardiothorac Vasc Anesth 1999;13:532-537.

22 Slinger P: The clinical use of right-sided double-lumen tubes. Can J Anaesth 2010;57:293-300.

23 de Bellis M, Accardo R, Di Maio M, La Manna C, Rossi GB, Pace MC, Romano V, Rocco G: Is flexible bronchoscopy necessary to confirm the position of double-lumen tubes before thoracic surgery? Eur J Cardiothorac Surg 2011;40:912-916.

24 Heidegger T, Schnider TW: "awake" or "sedated": safe flexible bronchoscopic intubation of the difficult airway. ANESTH ANALG 2017;124:996-997.

25 Wei W, Tian M: Double-lumen tube intubation using video laryngoscopy causes a milder cardiovascular response compared to classic direct laryngoscopy. PAK J MED SCI 2016;32:35-39.

26 Liu TT, Li L, Wan L, Zhang CH, Yao WL: Videolaryngoscopy vs. Macintosh laryngoscopy for double-lumen tube intubation in thoracic surgery: a systematic review and meta-analysis. ANAESTHESIA 2018;73:997-1007.

27 Campos $\mathrm{JH}$ : Update on tracheobronchial anatomy and flexible fiberoptic bronchoscopy in thoracic anesthesia. Curr Opin Anaesthesiol 2009;22:4-10.

28 Cohen E: Double-lumen tube position should be confirmed by fiberoptic bronchoscopy. Curr Opin Anaesthesiol 2004;17:1-6.

29 Brodsky JB, Macario A, Mark JB: Tracheal diameter predicts double-lumen tube size: a method for selecting left double-lumen tubes. ANESTH ANALG 1996;82:861-864. 
30 Lee JW, Son JS, Choi JW, Han YJ, Lee JR: The comparison of the lengths and diameters of main bronchi measured from two-dimensional and three-dimensional images in the same patients. Korean $\mathrm{J}$ Anesthesiol 2014;66:189-194.

31 Shiqing L, Wenxu Q, Yuqiang M, Youjing D: Predicting the size of a left double-lumen tube for asian women based on the combination of the diameters of the cricoid ring and left main bronchus: a randomized, prospective, controlled trial. ANESTH ANALG 2018

32 Shiqing L, Wenxu Q, Jin Z, Youjing D: The combination of diameters of cricoid ring and left main bronchus for selecting the "best fit" double-lumen tube. J Cardiothorac Vasc Anesth 2018;32:869-876.

33 Seymour AH, Prakash N: A cadaver study to measure the adult glottis and subglottis: defining a problem associated with the use of double-lumen tubes. J Cardiothorac Vasc Anesth 2002;16:196-198.

34 Benumof JL, Partridge BL, Salvatierra C, Keating J: Margin of safety in positioning modern double-lumen endotracheal tubes. ANESTHESIOLOGY 1987;67:729-738.

$35 \mathrm{Kim}$ JH, Park SH, Han SH, Nahm FS, Jung CK, Kim KM: The distance between the carina and the distal margin of the right upper lobe orifice measured by computerised tomography as a guide to rightsided double-lumen endobronchial tube use. ANAESTHESIA 2013;68:700-705.

36 Ehrenfeld JM, Mulvoy W, Sandberg WS: Performance comparison of right- and left-sided doublelumen tubes among infrequent users. J Cardiothorac Vasc Anesth 2010;24:598-601.

37 Lu XH, Zhang XM, Liu SL, Jiang FQ, Ding RX, Bin X, Jie Y, Gou XJ: Clinical research on prevention and treatment of respiratory tract complications with acupoint application after operation under general anesthesia. J CRANIOFAC SURG 2019;30:e85-e92.

\section{Figures}



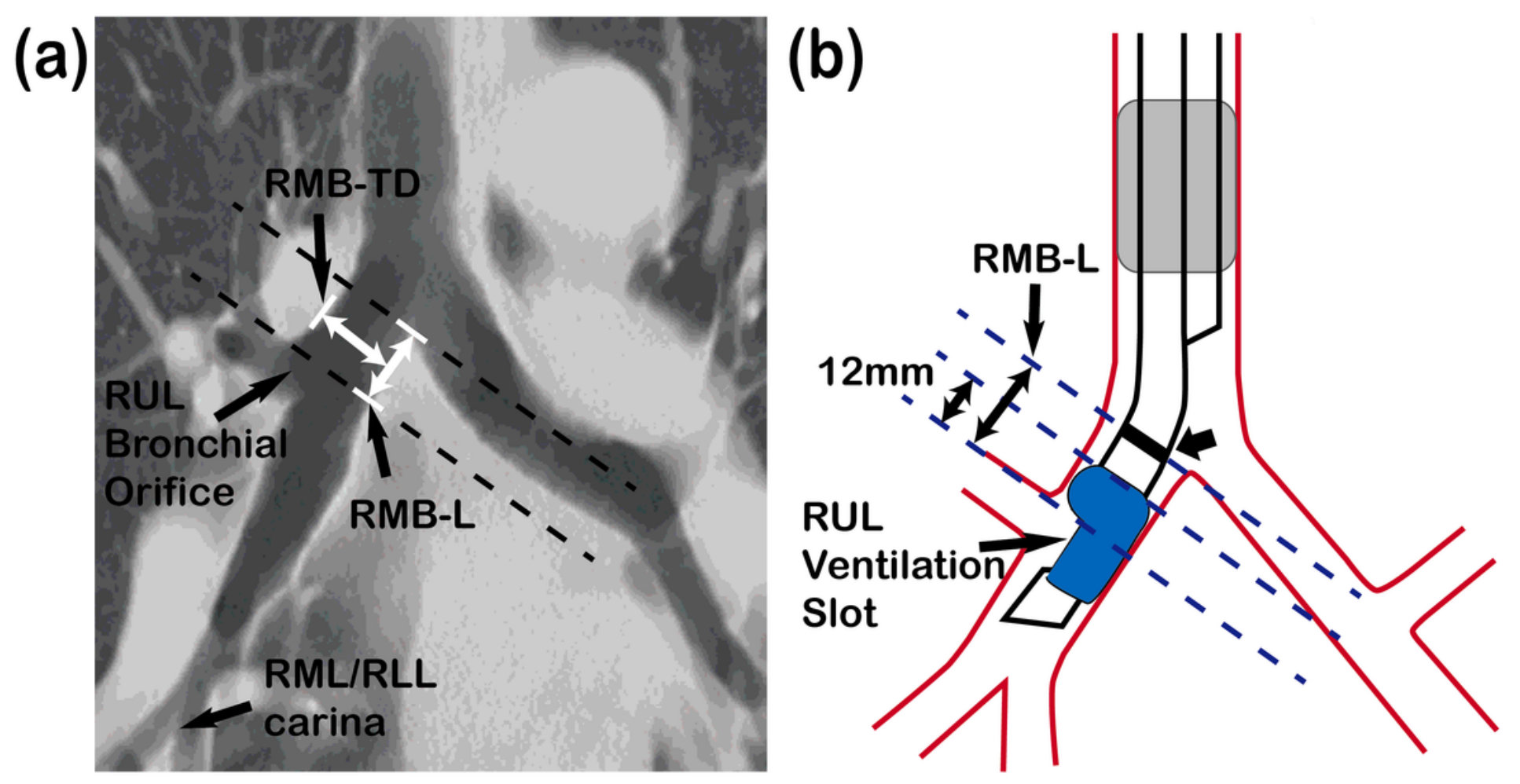

Figure 1

Supplemental digital fig 2. The modification and application of a domestic RDLT similar to our method. (a) FVB view via the tracheal lumen of RDLT. The tracheal carina (arrowhead) and the black maker (long arrow) on the right wall of the tube can be seen. (b) The modification of Mallinckrodt right-sided DLT. Note that two self-made markers were drawn on the top of the tube and above the bronchial cuff respectively. (c) FVB view via the distal end of the bronchial lumen of RDLT. Guide the front edge of the black marker to align with the RML/RLL carina (arrowhead). FVB, flexible video bronchoscope; RDLT, right-sided double-lumen tube; RMB-AD, right main bronchus - anteroposterior diameter; RML/RLL carina, right middle lobe /right lower lobe bronchus carina. 

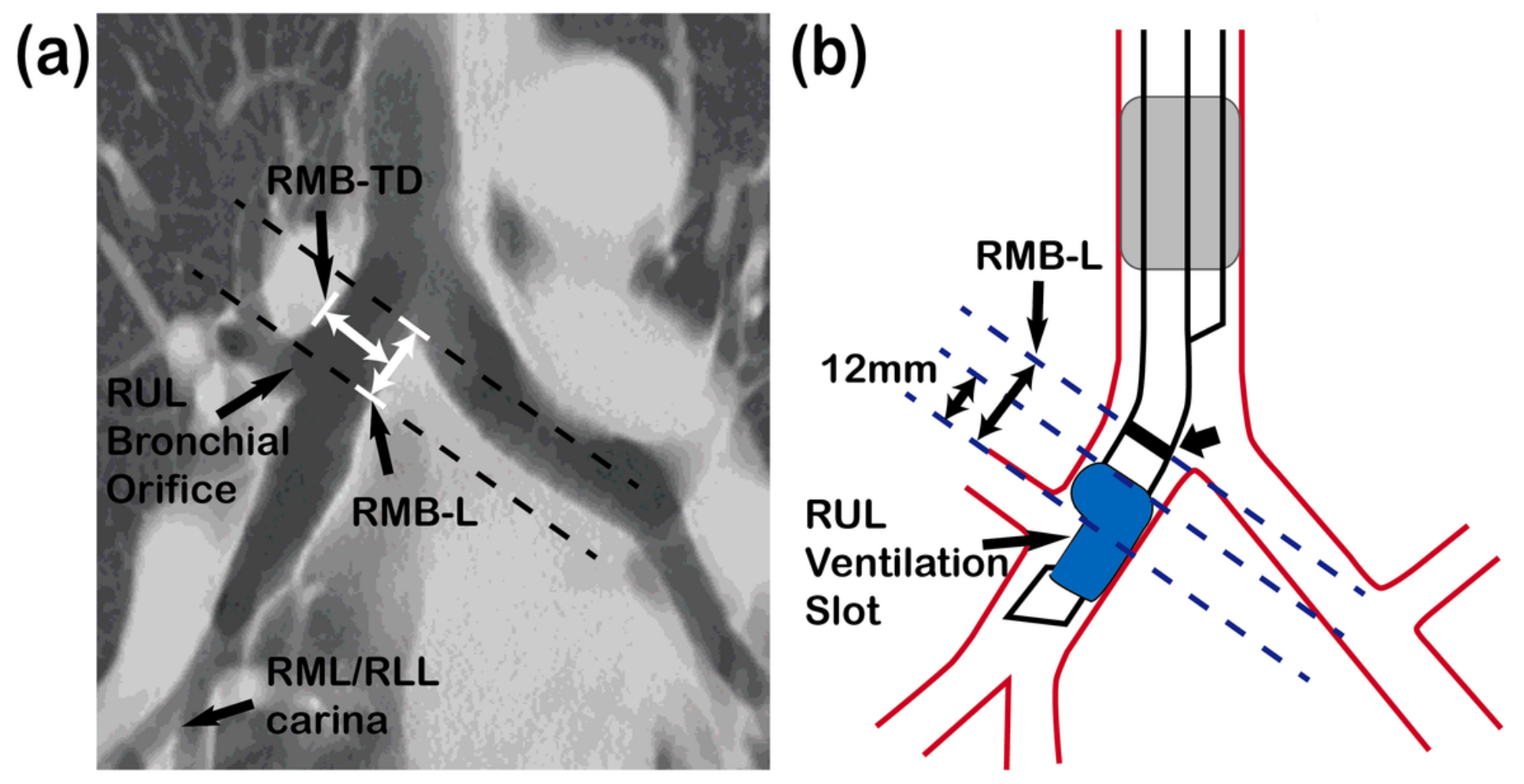

Figure 1

Supplemental digital fig 2. The modification and application of a domestic RDLT similar to our method. (a) FVB view via the tracheal lumen of RDLT. The tracheal carina (arrowhead) and the black maker (long arrow) on the right wall of the tube can be seen. (b) The modification of Mallinckrodt right-sided DLT. Note that two self-made markers were drawn on the top of the tube and above the bronchial cuff respectively. (c) FVB view via the distal end of the bronchial lumen of RDLT. Guide the front edge of the black marker to align with the RML/RLL carina (arrowhead). FVB, flexible video bronchoscope; RDLT, right-sided double-lumen tube; RMB-AD, right main bronchus - anteroposterior diameter; RML/RLL carina, right middle lobe /right lower lobe bronchus carina. 

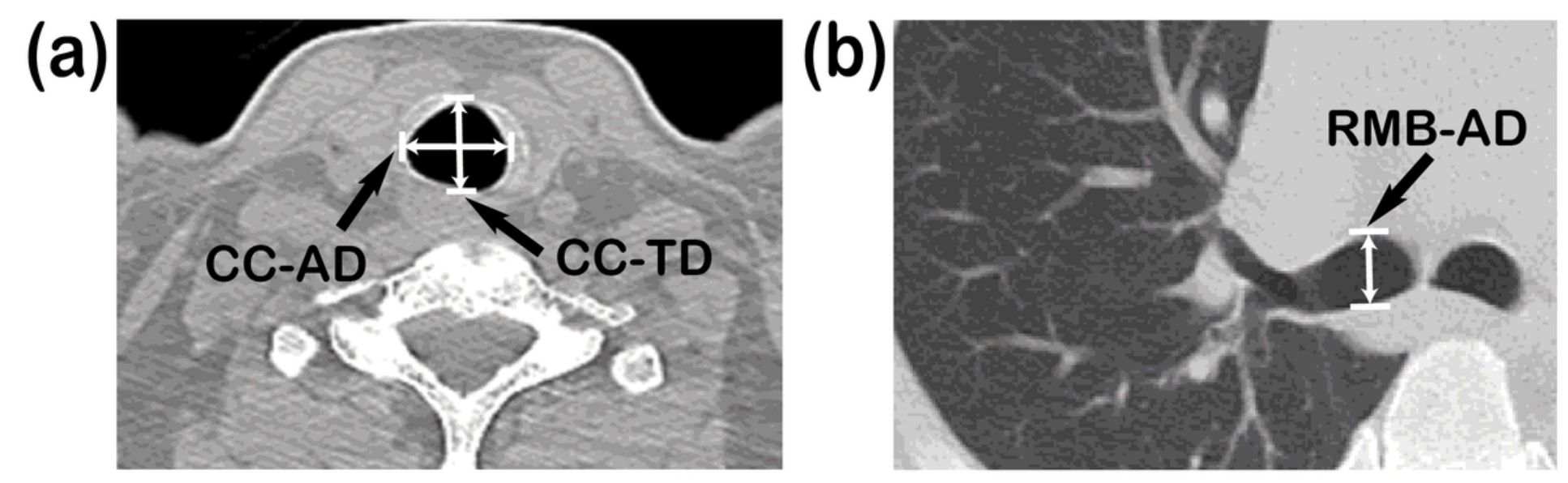

(c)

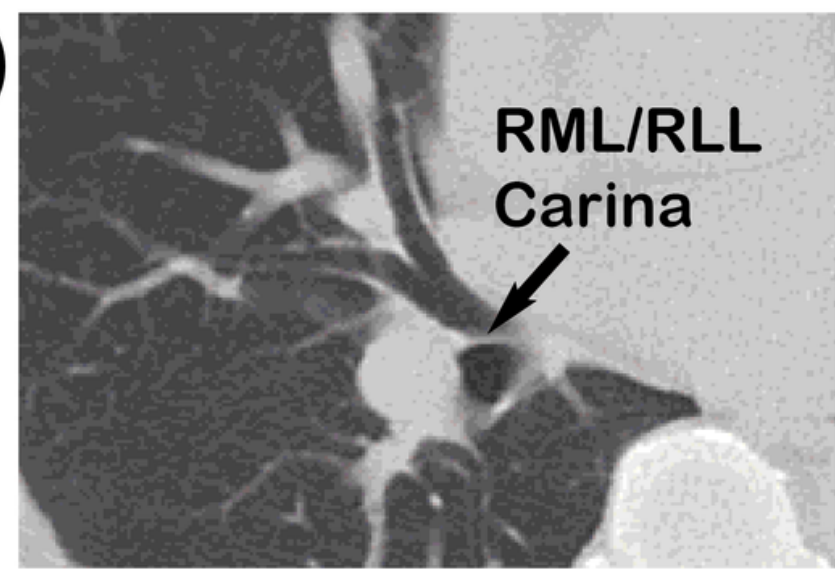

(d)

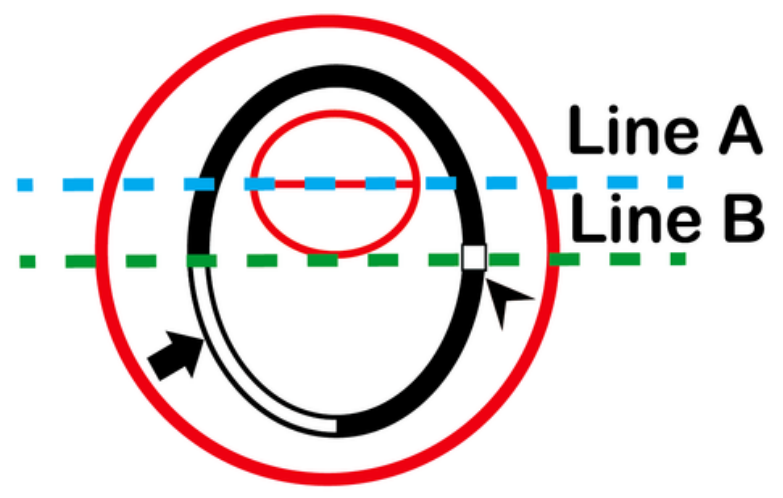

Figure 2

CT Measurements in the lung window and schematic diagram of the optimal position of RDLT. (a) CT Measurements of CC-TD and CC-AD in the lung window in cricoid cartilage plane. (b) CT Measurements of RMB-AD in the lung window in the RUL bronchial orifice plane. (c) RML/RLL carina on the tracheal window. (d) Schematic diagram of the optimal position of RDLT and Right main bronchus from the view of the tip of the bronchial lumen. The arrow indicates the position of the X-ray white line marker in the different parts of the RDLT. The round-tipped arrow indicates the position of the X-ray indicator at the tip of the tube. Line $A$ (blue) indicates the line crossing the RML/RLL carina. Line B (green) indicates the line between the white marker and the X-ray indicator. CC-AD, cricoid cartilage - anteroposterior diameter; CCTD, cricoid cartilage - transverse diameter; RDLT, right-sided double-lumen tube; RMB-AD, right main bronchus - anteroposterior diameter; RML/RLL carina, right middle lobe /right lower lobe bronchus carina. 

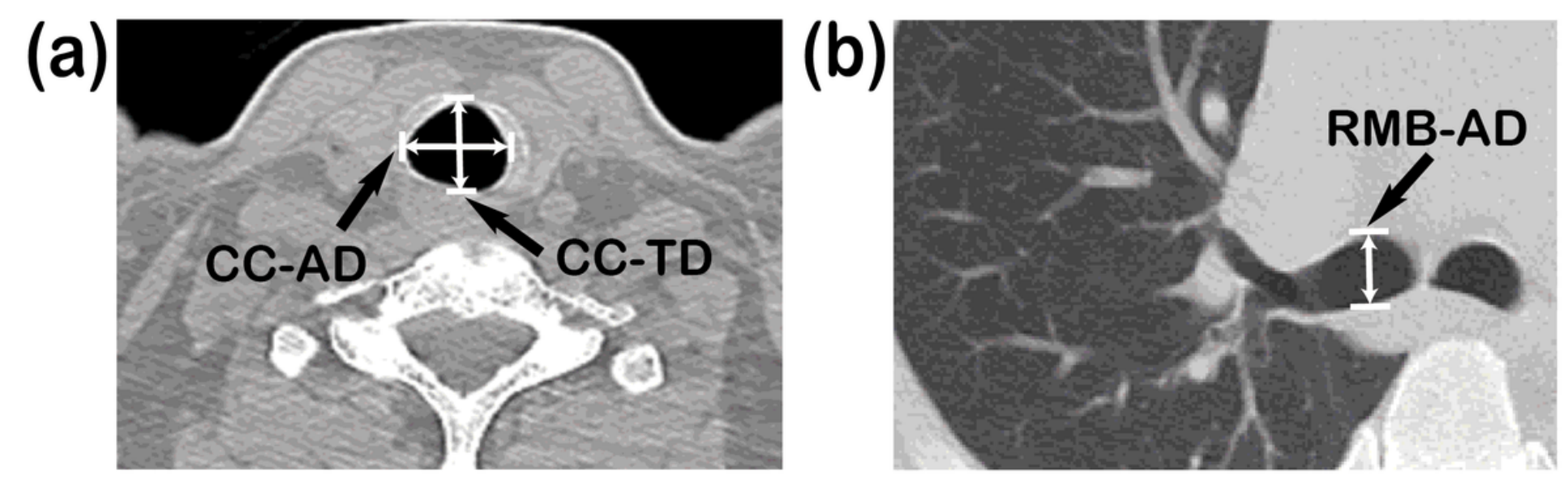

(c)

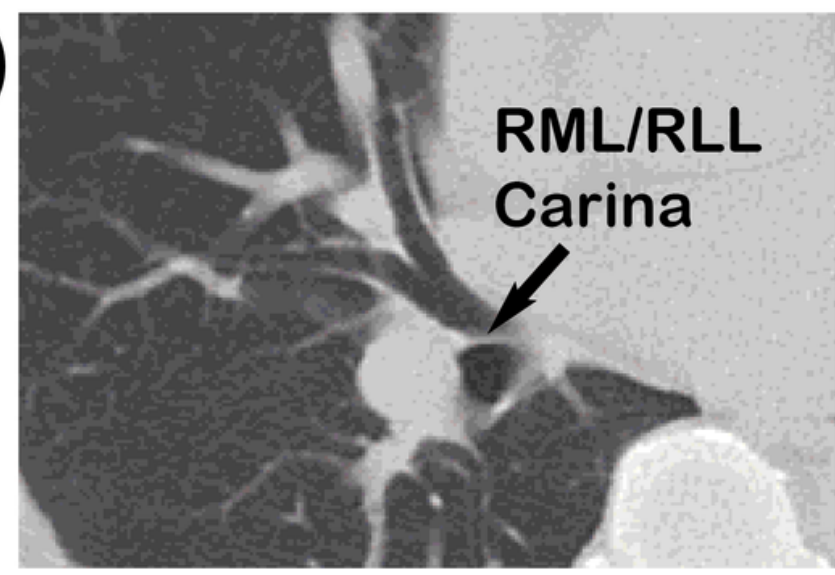

(d)

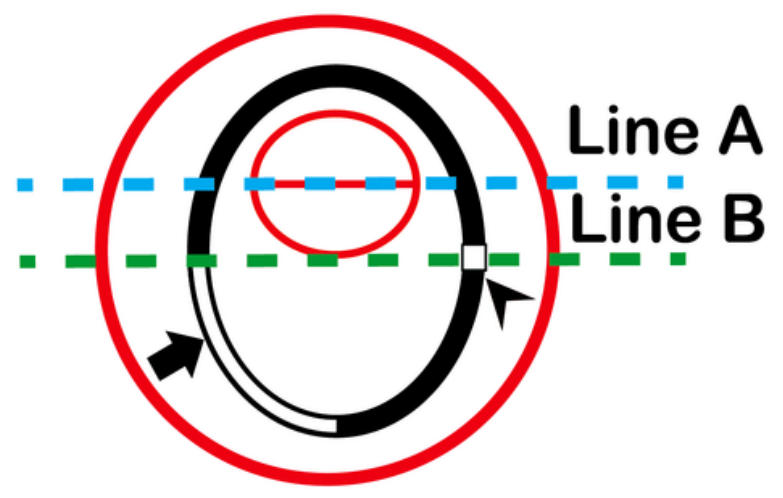

Figure 2

CT Measurements in the lung window and schematic diagram of the optimal position of RDLT. (a) CT Measurements of CC-TD and CC-AD in the lung window in cricoid cartilage plane. (b) CT Measurements of RMB-AD in the lung window in the RUL bronchial orifice plane. (c) RML/RLL carina on the tracheal window. (d) Schematic diagram of the optimal position of RDLT and Right main bronchus from the view of the tip of the bronchial lumen. The arrow indicates the position of the X-ray white line marker in the different parts of the RDLT. The round-tipped arrow indicates the position of the X-ray indicator at the tip of the tube. Line $A$ (blue) indicates the line crossing the RML/RLL carina. Line B (green) indicates the line between the white marker and the X-ray indicator. CC-AD, cricoid cartilage - anteroposterior diameter; CCTD, cricoid cartilage - transverse diameter; RDLT, right-sided double-lumen tube; RMB-AD, right main bronchus - anteroposterior diameter; RML/RLL carina, right middle lobe /right lower lobe bronchus carina. 


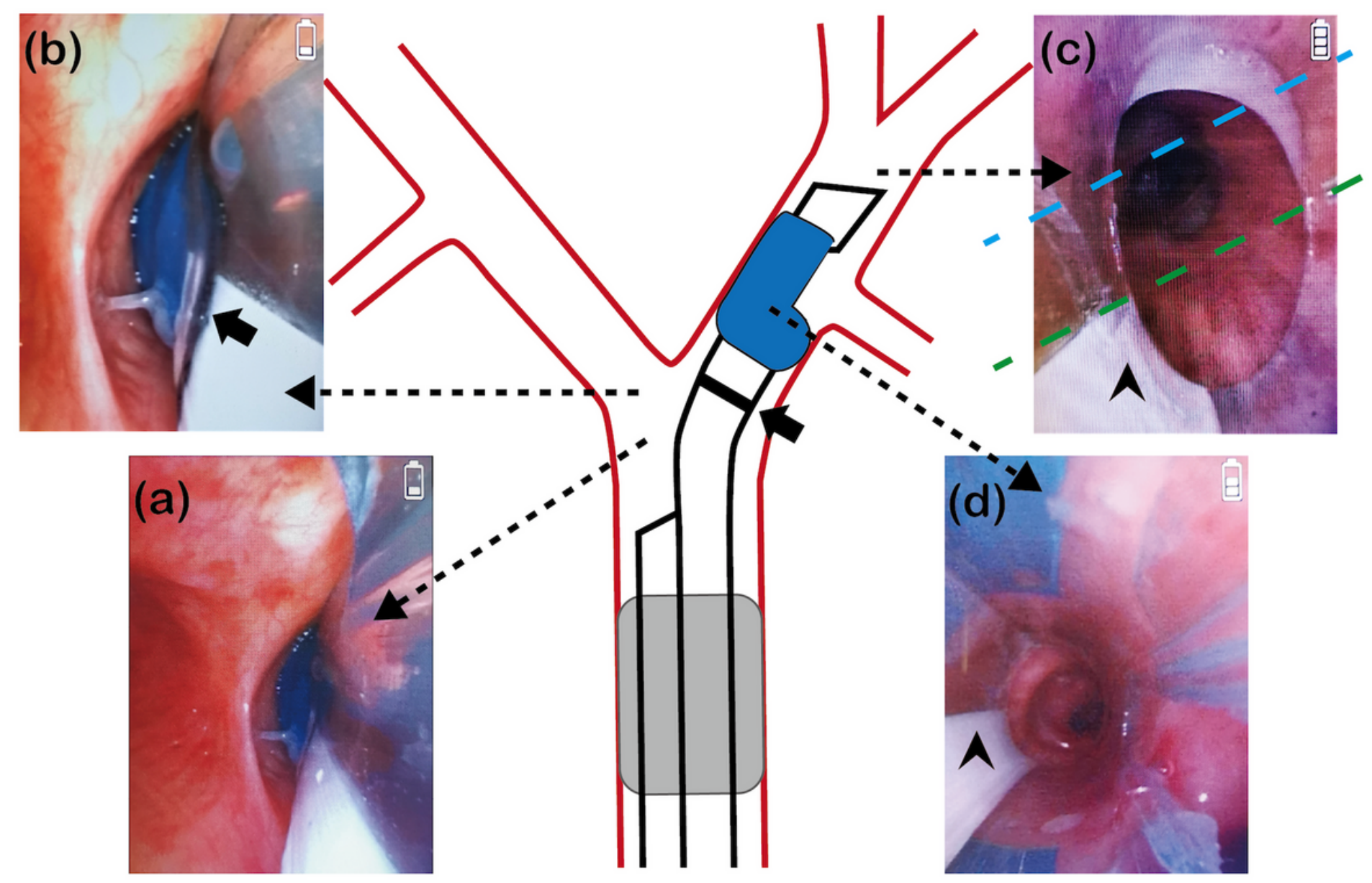

Figure 3

FVB-guided intubation of an RDLT. (a) FVB view via the tracheal lumen of RDLT. The tracheal carina and the black maker (long arrow) on the right wall of the tube can be seen. The tube is in too deep. (b) FVB view via the tracheal lumen of RDLT. Guide the black marker to align with the carina to achieve the best depth. (c) FVB view via the distal end of the bronchial lumen of RDLT. Guide the line between the white marker and X-ray indicator (triangle) to align with the RML/RLL carina (round tipped arrow). (d) FVB view via the bronchial lumen of RDLT. Withdraw the FVB and look for the ventilation slot on the opposite side of the white line marker. Through the slit of the ventilation slot, examine the alignment of the RUL ventilation slot and RUL bronchial orifice. Blue line indicates the line crossing the RML/RLL carina. Green line indicates he line between the white marker and the X-ray indicator. FVB, flexible video bronchoscope; RDLT, right-sided double-lumen tube; RMB-AD, right main bronchus - anteroposterior diameter; RML/RLL carina, right middle lobe /right lower lobe bronchus carina. 


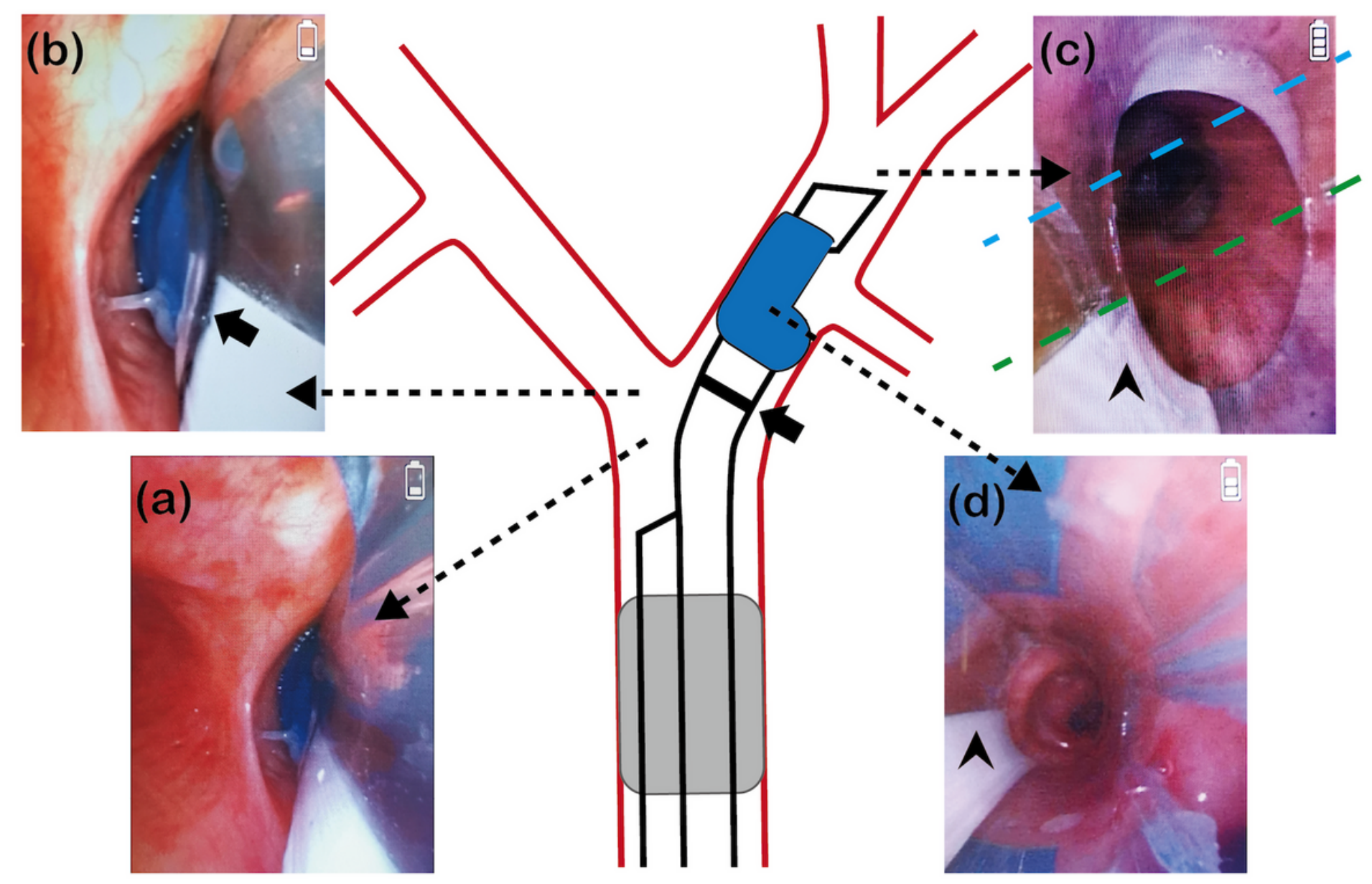

Figure 3

FVB-guided intubation of an RDLT. (a) FVB view via the tracheal lumen of RDLT. The tracheal carina and the black maker (long arrow) on the right wall of the tube can be seen. The tube is in too deep. (b) FVB view via the tracheal lumen of RDLT. Guide the black marker to align with the carina to achieve the best depth. (c) FVB view via the distal end of the bronchial lumen of RDLT. Guide the line between the white marker and X-ray indicator (triangle) to align with the RML/RLL carina (round tipped arrow). (d) FVB view via the bronchial lumen of RDLT. Withdraw the FVB and look for the ventilation slot on the opposite side of the white line marker. Through the slit of the ventilation slot, examine the alignment of the RUL ventilation slot and RUL bronchial orifice. Blue line indicates the line crossing the RML/RLL carina. Green line indicates he line between the white marker and the X-ray indicator. FVB, flexible video bronchoscope; RDLT, right-sided double-lumen tube; RMB-AD, right main bronchus - anteroposterior diameter; RML/RLL carina, right middle lobe /right lower lobe bronchus carina. 


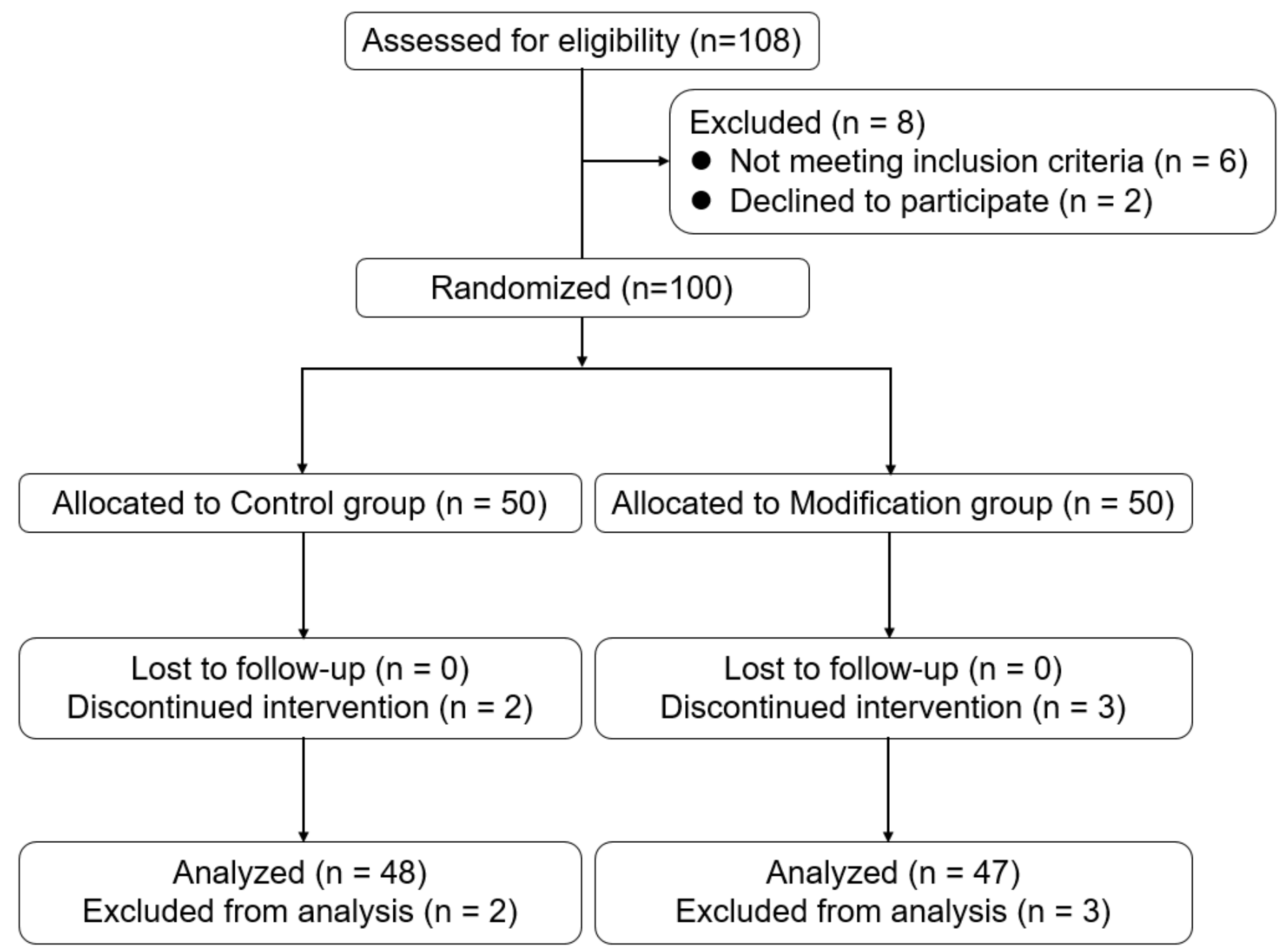

Figure 4

Flow diagram. 


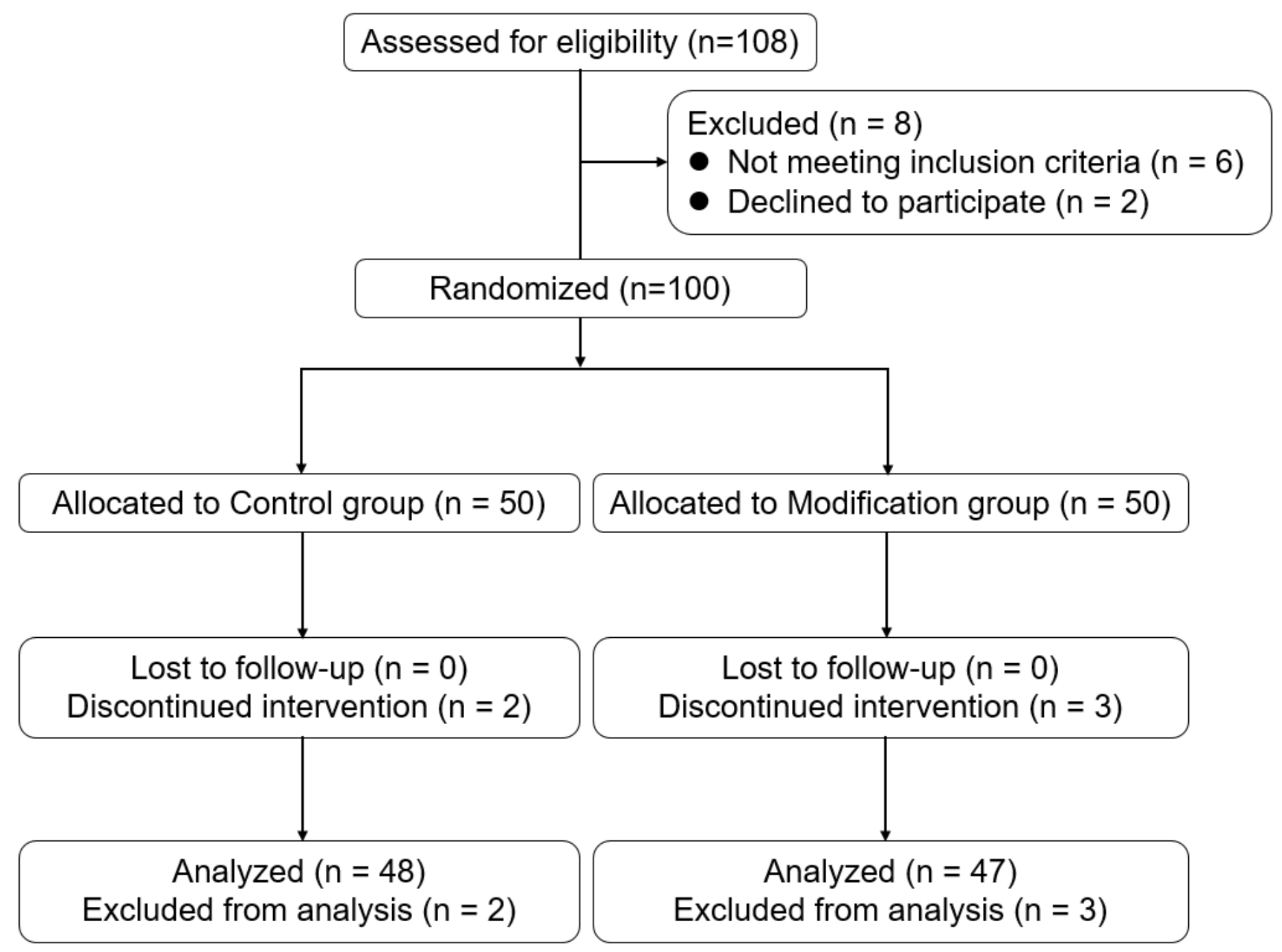

Figure 4

Flow diagram.

\section{Supplementary Files}

This is a list of supplementary files associated with this preprint. Click to download.

- CONSORT2010ChecklistBMC.pdf

- CONSORT2010ChecklistBMC.pdf

- Supplementalfigure1.tif

- Supplementalfigure1.tif

- Supplementalfigure2.tif

- Supplementalfigure2.tif 